\section{Mutaciones y polimorfismos mitocondriales en un paciente mexicano con cáncer de próstata}

\author{
Campos-García V, Vázquez-Morfín C, Alvarado-Núñez I, Ramírez-Noguera \\ $\mathrm{P}$, Montiel-Sosa $\mathrm{F}$
}

\section{Resumen}

ANTECEDENTES: la etiología genética del cáncer de próstata, la neoplasia más común en los países occidentales, es compleja y la interacción de los genes con los factores ambientales de la enfermedad se ha vuelto de interés en los últimos años. Los estudios de las mutaciones somáticas en el ADN mitocondrial se han convertido en un aspecto importante de la investigación del cáncer porque estas variaciones pueden tener consecuencias funcionales y servir como biomarcadores para la detección y evolución tumoral prostática.

CASO CLÍNICO: reporte de la secuenciación bidireccional del mitogenoma $(16,569 \mathrm{pb})$ de tejidos celulares, maligno y sano, proveniente de la resección transuretral de próstata de un paciente mexicano diagnosticado con cáncer de próstata. Gracias a las herramientas bioinformáticas especializadas, MITOMAP y Mitlmpact CSS Bioinformatics Lab, se localizaron las primeras mutaciones susceptibles al cáncer de próstata, T489C, G6261A, C12705T y C16223T. Otras mutaciones probables encontradas fueron: C13478G y G12009A (aún no descritas en la bibliografía médica). Todas las variaciones estudiadas fueron homoplásmicas. Se identificó el haplogrupo A2 mitocondrial del paciente. En la actualidad, se estudia la frecuencia de haplogrupos mitocondriales en poblaciones europeas y asiáticas asociadas al cáncer de próstata; sin embargo, se carece de estudios similares en la población mexicana.

CONCLUSIÓN: las mutaciones somáticas en el genoma mitocondrial son eventos frecuentes en el cáncer de próstata que se constituyen en una herramienta complementaria a los estudios tradicionales de diagnóstico de este cáncer.

PALABRAS CLAVE: cáncer de próstata, haplogrupos mitocondriales, mutaciones en el mtDNA, bioinformática, resección transuretral de próstata, mutaciones homoplásmicas.
Departamento de Ciencias Biológicas, Facultad de Estudios Superiores Cuautitlán, Universidad Nacional Autónoma de México, México.

Recibido: abril 2016

Aceptado: enero 2017

Correspondencia

Dr. Francisco Montiel Sosa

fmontiel_sosa@yahoo.com.mx

Este artículo debe citarse como

Campos-García V, Vázquez-Morfín C, AlvaradoNúñez I, Ramírez-Noguera P, Montiel-Sosa F. Mutaciones y polimorfismos mitocondriales en un paciente mexicano con cáncer de próstata. Rev Mex Urol. 2017 ene;77(1):61-70. 


\section{Mitochondrial mutations and polymorphisms in a Mexican prostate cancer patient}

Campos-García V, Vázquez-Morfín C, Alvarado-Núñez I, Ramírez-Noguera $\mathrm{P}$, Montiel-Sosa $\mathrm{F}$

\begin{abstract}
BACKGROUND: The genetic etiology of prostate cancer, the most common neoplasia in the western countries, is complex, and the interaction of disease genes with environmental factors has been of interest in recent years. Studies on somatic mutations in mitochondrial DNA (mtDNA) have become important in cancer research because these variations can have functional consequences and serve as biosensors for prostate tumor detection and progression.
\end{abstract}

CLINICAL CASE: In the present study we bidirectionally sequenced the mitochondrial genome (16,569 pb) of both malignant and healthy tissue from the transurethral resection of the prostate of a Mexican patient diagnosed with prostate cancer. Thanks to the specialized bioinformatic tools, MITOMAP and Mitlmpact, CSS Bioinformatics Lab, the first prostate cancer mutation candidates - T489C, G6261A, $\mathrm{C} 12705 \mathrm{~T}$, and C16223T - were located. Other probable mutations found were: C13478G and G12009A (not yet described in the medical literature). All the variations studied were homoplasmic. Likewise, the mitochondrial A2 haplogroup of the patient was identified. Mitochondrial haplogroup frequency is currently being studied in European and Asian populations associated with prostate cancer. However, there are no similar studies conducted on a Mexican population.

CONCLUSION: In short, somatic mutations in the mitochondrial genome are frequent events in prostate cancer, thus becoming a complementary tool in regard to the traditional prostate cancer diagnostic studies.

KEY WORDS: Prostate cancer; Mitochondrial haplogroups, mtDNA mutations; Bioinformatics; Transurethral resection of the prostate; Homoplasmic mutations
Departamento de Ciencias Biológicas Facultad de Estudios Superiores Cuautitlán, Universidad Nacional Autónoma de México, México.

Correspondence Dr. Francisco Montiel Sosa fmontiel_sosa@yahoo.com.mx

\section{ANTECEDENTES}

En México y en otros países occidentales, el cáncer de próstata se ha convertido en un problema de salud pública, porque la tasa de mortalidad anual es de 121.57 por cada 100 mil hombres, lo que representa $8.1 \%$ de las defunciones en la población masculina de 60 años. ${ }^{1}$ Ante esto, diversas investigaciones se han dedicado a identificar marcadores que pronostican la fase 
primaria y agresiva del cáncer de próstata. ${ }^{2}$ Éste es el caso de las mutaciones encontradas, durante las últimas décadas, en el genoma mitocondrial. ${ }^{3}$

La mitocondria participa en el proceso de carcinogénesis, por su papel vital en la producción de energía ${ }^{4} y$ apoptosis. ${ }^{5}$ El genoma mitocondrial exhibe una alta tasa de mutación que se acumula en mutaciones somáticas y pérdidas en tejidos tumorales. ${ }^{6-7}$ La evolución del ADN mitocondrial se caracteriza por la identificación de haplogrupos, grupos de genealogías evolutivas estrechamente relacionadas que definen ciertos polimorfismos. ${ }^{8}$ Debido a que estos haplogrupos, con polimorfismos específicos son ampliamente neutrales se ha estudiado su presunta asociación con el riesgo de cáncer de próstata. ${ }^{9}$ En Europa no se encontró la influencia del haplogrupo mitocondrial con el cáncer de próstata, ${ }^{10} \mathrm{ni}$ en la población asiática; ${ }^{11}$ sin embargo, se ha reportado que puede ser un factor de riesgo en la población de América del Norte. ${ }^{12}$

Otra característica del ADN mitocondrial es la aparición de la heteroplasmia, que se define como la coexistencia de dos o más poblaciones de moléculas de ADN mitocondrial con una ligera diferencia en la composición de nucleótidos en una célula o tejido. Se cree que la identificación de bajas concentraciones de heteroplasmia puede permitir un diagnóstico temprano y la vigilancia de la evolución al cáncer de próstata. ${ }^{13}$

En la actualidad, la influencia del ADN mitocondrial en el origen y evolución del cáncer de próstata no ha sido entendida completamente. Sin embargo, existen estudios que apoyan un papel activo del ADN mitocondrial en la tumorogénesis prostática. ${ }^{14}$

Se secuenció bidireccionalmente el mitogenoma completo en tejidos, malignos y sanos, provenientes de una resección transuretral de próstata, con grado $4+4$ Gleason, suma de Gleason 8 . Las secuencias mitogenómicas se analizaron en la base de datos pública MITOMAP. Con esta estrategia y las bajas concentraciones de heteroplasmia, que en este caso no se observaron, sino que las moléculas del ADN mitocondrial en ambos tejidos fueron homoplásmicas, puedan ser una herramienta para identificar,en una fase temprana, la tumorogénesis y evolución a cáncer de próstata. El objetivo de esta investigación es identificar las mutaciones somáticas en el ADN mitocondrial asociadas al cáncer de próstata.

\section{CASO CLÍNICO}

Paciente de 70 años de edad, con antígeno prostático específico, APE, $20 \mathrm{ng} / \mathrm{mL}$. Próstata de $2.0 \times 2.0 \mathrm{~cm}$, de consistencia pétrea, con un nódulo palpable en el lóbulo izquierdo. La biopsia prostática y endoscopia con resección transuretral reportaron: adenocarcinoma poco diferenciado grado $4+4$ de Gleason en $90 \%$ del material estudiado. La inmunohistoquímica informó: citoqueratina B/1B positiva y fosfatasa ácida prostática positiva. Se practicaron estudios de extensión con tomografía axial computada más rastreo óseo gammagráfico, en donde se apreció francamente la metástasis ganglionar y ósea, motivo por lo que fue necesaria la propuesta de tratamiento paliativo con bloqueo androgénico completo con análogos agonistas LH-RH y anti andrógenos, tipo bicalutamida.

\section{Colección de las muestras y extracción del ADN} genómico. Posterior a la resección transuretral de próstata y el reporte histopatológico, se recolectaron los tejidos, maligno y sano. Las muestras se resguardaron, primeramente, a $-4^{\circ} \mathrm{C}$ después de la obtención por un promedio máximo de 3 horas y después a $-20^{\circ} \mathrm{C}$ en tubos Falcon ${ }^{\circledR}$ con solución fisiológica. La extracción de ADN genómico total se realizó según el protocolo Quiagen ${ }^{\circledR}$ DNeasy Blood \& Tissue Kit mediante el método de cromatografía de adsorción (minicolumnas de 
sílice bajo condiciones iónicas controladas). Para medir la concentración $[\mathrm{ng} / \mathrm{mL}$ ] y pureza (relación 260/280) del ADN se utilizó un espectrofotómetro ultravioleta, NanoDrop ND-1000 ${ }^{\circledR}$, en los límites del espectro de absorción de 200 a 350 nm (UV) de los ácidos nucleicos.

\section{Amplificación del mitogenoma y secuenciación}

Mediante reacción en cadena de la polimerasa de punto final (PCR) se amplificó el mitogenoma completo de las muestras de ADN, que se efectuó en $12.5 \mu \mathrm{L}$ de volumen final. La mezcla contenía $1 \mu \mathrm{L}$ del ADN plantilla, $0.5 \mu \mathrm{L}$ a 25 $\mu \mathrm{M}$ de cada primer frontal y reverso, $6.25 \mu \mathrm{L}$ de GoTaq ${ }^{\circledR}$ G2 Hot Start Colorless Master Mix, Promega (GoTaq ${ }^{\circledR} \mathrm{G} 2$ Hot Start DNA Polimerasa, Buffer de reacción ( $\mathrm{pH} 8.5), 400 \mu \mathrm{M}$ dATP, $400 \mu \mathrm{M} \mathrm{dGTP}, 400 \mu \mathrm{M}$ dCTP, $400 \mu \mathrm{M}$ dTTP y $4 \mathrm{mM}$ $\mathrm{MgCl} 2$ ) y $4.25 \mu \mathrm{L}$ de $\mathrm{H}_{2} \mathrm{O} 18.2 \Omega$-cm (Milli-Q ${ }^{\circledR}$, Millipore $\mathrm{Co}$ ). Las condiciones de PCR fueron: temperatura inicial de desnaturalización a $95^{\circ} \mathrm{C}$ durante dos minutos, seguido de 35 ciclos a $95^{\circ} \mathrm{C}$ por espacio de 30 segundos, a $60^{\circ} \mathrm{C}$ durante 45 segundos y un minuto a $72^{\circ} \mathrm{C}$ y una extensión final de $72^{\circ} \mathrm{C}$ durante 5 minutos. La amplificación completa del ADN mitocondrial se efectuó en el termociclador Apollo ${ }^{\circledR}$ ATC- 401 (GX design engineers, RU). Para visualizar los productos de PCR del ADN mitocondrial se realizó una electroforesis en gel de agarosa (CAS No. 9012-36-6, Sigma Aldrich ${ }^{\circledR}$ ) al $0.7 \%$ teñida con bromuro de etidio $0.5 \mathrm{mg} / \mathrm{mL}$, (CAS No. 1239-45-8, Sigma Aldrich $^{\circledR}$ ) en amortiguador TAE 1X (T6025, Sigma Aldrich $\left.^{\circledR}\right)$, programándose la fuente de poder (Bio-Rad $^{\circledR}$ Powerpac 200), a 70 volts $40 \mathrm{~min}$. Posteriormente, los productos de PCR se purificaron según el protocolo ExoSAP-IT ${ }^{\circledR}$ PCR Product Cleanup, Affymetrix. Se aplicó la técnica de secuenciación bidireccional automática mediante electroforesis capilar (Soporte polímero DT3130 POP7, química BigDye ${ }^{\circledR}$ Terminator v3.1 Cycle Sequencing Kit, Applied Biosystems), con los cebadores diseñados para el mitogenoma humano
(Homo sapiens sapiens), en un secuenciador de 16 capilares ABI Prism 3130XL Genetic Analyzer (Applied Biosystems, EUA).

\section{Análisis de datos}

Se efectuó un análisis completo del ADN mitocondrial por medio de la comparación de las secuencias experimentales con los datos de otros estudios, reportados en la base de datos del GenBank del Centro Nacional para la Información Biotecnológica ( $\mathrm{NCBI}$, por sus siglas en inglés) de Estados Unidos. Las secuencias disponibles del mitogenoma humano (Homo sapiens sapiens), Homo sapiens isolate mitochondrion, complete genome Accession: KX228162.1-193.1, Homo sapiens isolate control región, partial sequence Accession KC807000.1 -164.1; la secuencia control de referencia fue, NC_012920 (The revised Cambridge Reference Sequence rCRS). Para eliminar ambigüedades, las secuencias se editaron manualmente, con el programa Geneious 4.8.2 mediante un alineamiento múltiple de las secuencias muestreadas y referidas en el Genbank mediante el programa MUSCLE 3.6 con los parámetros convencionales. El alineamiento es una etapa decisiva para los análisis filogenéticos, porque es el paso donde se define la homología de los sitios. El objetivo fue asegurar que cada sitio elegido fuese homólogo; es decir, que se adquirió de un ancestro común y que contiene información filogenética relevante, para identificar correctamente el haplogrupo de las secuencias experimentales con las de referencia. Con el programa bioinformático MITOMAP se localizaron las mutaciones y polimorfismos de las secuencias experimentales y la secuencia de referencia, NC_012920, reportadas en esta base de datos. La herramienta bioinformática Mitlmpact CSS Bioinformatics Lab se utilizó para pronosticar la patogenicidad de los cambios de nucleótidos que causan sustituciones no sinónimas en los genes codificantes para proteínas mitocondriales. 


\section{DISCUSIÓN}

Se analizó el mitogenoma completo de un paciente con diagnóstico de cáncer de próstata. Las muestras se obtuvieron mediante resección transuretral de próstata que se dividieron en tejido sano (C-) y tejido maligno $(C+)$, según el reporte de inmunohistoquímica (citoqueratina B/1B + y fosfatasa ácida prostática + ).

Está confirmado, por observaciones previas de Jerónimo y su equipo, que las mutaciones en el ADN mitocondrial son comunes en el cáncer de próstata. Mediante el análisis de las secuencias de ADN y programas bioinformáticos especializados (MITOMAP y Mitlmpact 2) se encontraron cuatro mutaciones asociadas al cáncer de próstata: T489C, C12705T, G6261A, C16223T, las primeras reportadas en México. Cuadro 1 y Figura 1.

En la comprensión de nuestra investigación del comportamiento del mitogenoma asociado al cáncer de próstata, no observamos una diferencia significativa entre la tasa de mutaciones del tejido sano (C-) y el tejido maligno (C+). Tal es el caso de las mutaciones T489C, G6261A, C16223T, que no sólo se limitaron al tejido maligno sino también al sano. Esto se debe a que las alteraciones moleculares que pueden indicar la existencia de un tumor o contribuir a su crecimiento, tienen lugar mucho antes de que las variaciones morfológicas malignas sean visibles, incluso en reportes histopatológicos. En este sentido, la existencia de un tumor puede ejercer un "efecto de campo" que conduce a los cambios moleculares que aparecen en el tejido histológicamente normal adyacente al tumor, este efecto se demostró recientemente en perfiles de expresión génica del cáncer de próstata. ${ }^{15-17}$

La mutación C12705T, localizada sólo en el tejido maligno $(\mathrm{C}+)$, en el locus mitocondrial MT-ND5, genera un desequilibrio en el proceso de la fosforilación oxidativa. Jerónimo y su grupo

Cuadro 1. Mutaciones y polimorfismos del mitogenoma de un paciente mexicano con cáncer de próstata. (Continiúa en la siguiente página)

\begin{tabular}{|c|c|c|c|}
\hline Posición & Locus & Descripción & Referencias \\
\hline 489 & MT-DLOOP & $\begin{array}{l}\text { Cáncer de próstata } \\
\text { homoplásmica }(+)\end{array}$ & https://www.mitomap.org/bin/view.pl/Main/SearchAllele \\
\hline 663 & MT-RNR1 & $\begin{array}{c}\text { Riesgo de aterosclerosis coronaria/ } \\
\text { N-non R subgrupo: } \\
\text { Haplogrupo N subgrupo A }\end{array}$ & http://www.mitomap.org/MITOMAP/HaplogroupMarkers \\
\hline 750 & MT-RNR1 & $\mathrm{H} 2 \mathrm{a}$ & Van Oven, 2010 \\
\hline 1,438 & MT-RNR1 & Haplogrupo H & Van Oven, 2010 \\
\hline 1,736 & MT-RNR2 & $\begin{array}{c}\text { Haplogrupo } \\
\text { A }\end{array}$ & http://www.mitomap.org/MITOMAP/HaplogroupMarkers \\
\hline 2,706 & MT-RNR2 & $\begin{array}{l}\text { Haplogrupo } \\
\text { LO, D4b, F1 }\end{array}$ & http://www.mitomap.org/MITOMAP/HaplogroupMarkers \\
\hline 3,107 & MT-RNR2 & $\begin{array}{c}\text { Haplogrupo } \\
\text { H2a2a1 }\end{array}$ & http://www.mitomap.org/MITOMAP/HaplogroupMarkers \\
\hline 4,248 & MT-ND1 & $\begin{array}{c}\text { Haplogrupo } \\
\text { D4a }\end{array}$ & http://www.mitomap.org/MITOMAP/HaplogroupMarkers \\
\hline 4,824 & MT-ND2 & Haplogrupo L1, D, N9, A & http://www.mitomap.org/MITOMAP/HaplogroupMarkers \\
\hline
\end{tabular}


Cuadro 1. Mutaciones y polimorfismos del mitogenoma de un paciente mexicano con cáncer de próstata. (Continuación)

\begin{tabular}{|c|c|c|c|}
\hline Posición & Locus & Descripción & Referencias \\
\hline 6,261 & MT-CO1 & Asociada a Cáncer de Próstata & $\begin{array}{l}\text { http://www.mitomap.org/bin/view.pl/Main/SearchAllele } \\
\text { Petros, J. A., Baumann, A. K., Ruiz-Pesini, E., Amin, M. } \\
\text { B., Sun, C. Q., Hall, J., Lim, S., Issa, M. M., Flanders, } \\
\text { W. D., Hosseini, S. H., Marshall, F. F., Wallace, D. C. } \\
\text { (2005) mtDNA mutations increase tumorigenicity in } \\
\text { prostate cancer Proceedings of the National Academy } \\
\text { of Sciences of the United States of America . } 102 \text { (3): } \\
719-724\end{array}$ \\
\hline 7,028 & MT-CO1 & $\begin{array}{l}\text { Haplogrupo } \\
\text { Q3 }\end{array}$ & http://www.mitomap.org/MITOMAP/HaplogroupMarkers \\
\hline 8,027 & MT-CO1 & Haplogrupo L0, L1, A2, R31 & http://www.mitomap.org/MITOMAP/HaplogroupMarkers \\
\hline 8,794 & MT-ATP6 & $\begin{array}{c}\text { C8794T } \\
\text { Homoplásmica }(+) \\
\text { Intolerancia al ejercicio y riesgo de } \\
\text { aterosclerosis coronaria }\end{array}$ & $\begin{array}{l}\text { http://www.mitomap.org/bin/view.pl/Main/SearchAllele } \\
\text { Sawabe, M., Tanaka, M., Chida, K., Arai, T., Nishigaki, } \\
\text { Y., Fuku, N., Mieno, M. N., Kuchiba, A., Tanaka, N. } \\
\text { (2011) Mitochondrial haplogroups A and M7a confer a } \\
\text { genetic risk for coronary atherosclerosis in the Japanese } \\
\text { elderly: an autopsy study of 1,536 patients Journal of } \\
\text { Athero }\end{array}$ \\
\hline 8,862 & MT-ATP6 & $\begin{array}{l}\text { A8862G } \\
\text { Epilepsia }\end{array}$ & $\begin{array}{l}\text { http://www.mitomap.org/bin/view.pl/MITOMAP/Variant- } \\
\text { SubmissionList }\end{array}$ \\
\hline 9,136 & MT-ATP6 & Haplogrupo LO & http://www.mitomap.org/MITOMAP/HaplogroupMarkers \\
\hline 9,300 & MT-CO3 & $\begin{array}{l}\text { Haplogrupo } \\
\text { LO/Miopatía }\end{array}$ & $\begin{array}{l}\text { http://www.mitomap.org/MITOMAP/HaplogroupMarkers } \\
\text { http://www.mitomap.org/bin/view.pl/MITOMAP/Variant- } \\
\text { SubmissionList }\end{array}$ \\
\hline 12,007 & MT-ND4 & Cáncer oral haplogrupo L2a & $\begin{array}{l}\text { http://www.mitomap.org/MITOMAP/HaplogroupMarkers } \\
\text { http://www.mitomap.org/bin/view.pl/MITOMAP/Variant- } \\
\text { SubmissionList }\end{array}$ \\
\hline 12,009 & MT-ND4 & $\begin{array}{l}\text { Mutación no descrita } \\
\text { Posible asociación con cáncer } \\
\text { de próstata }\end{array}$ & $\begin{array}{l}\text { http://bioinformatics.css-mendel.it/index.php/ } \\
\text { tools/2014-01-10-22-56-33/web-service }\end{array}$ \\
\hline 12,705 & MT-ND5 & $\begin{array}{c}\text { Cáncer de próstata } \\
\text { Homoplásmica }(+) \\
\text { C12705 } \\
\text { Haplogrupo R }\end{array}$ & $\begin{array}{l}\text { http://www.mitomap.org/MITOMAP/HaplogroupMarkers } \\
\text { http://www.mitomap.org/bin/view.pl/MITOMAP/Variant- } \\
\text { SubmissionList }\end{array}$ \\
\hline 13478 & C-G & $\begin{array}{l}\text { Posible asociación con } \\
\text { cáncer de próstata }\end{array}$ & $\begin{array}{l}\text { http://bioinformatics.css-mendel.it/index.php/ } \\
\text { tools/2014-01-10-22-56-33/web-service }\end{array}$ \\
\hline 14,766 & MIT-CYB & Haplogrupo L0, M2, I2 & $\begin{array}{l}\text { http://www.mitomap.org/MITOMAP/HaplogroupMarkers } \\
\text { http://www.mitomap.org/bin/view.pl/MITOMAP/Variant- } \\
\text { SubmissionList }\end{array}$ \\
\hline 15,172 & MT-CYB & Haplogrupo M1, M5, M10, D. & $\begin{array}{l}\text { http://www.mitomap.org/MITOMAP/HaplogroupMarkers } \\
\text { http://www.mitomap.org/bin/view.pl/MITOMAP/Variant- } \\
\text { SubmissionList }\end{array}$ \\
\hline 16,223 & MT-DLOOP & Tumor & https://www.mitomap.org/bin/view.pl/Main/SearchAllele \\
\hline
\end{tabular}




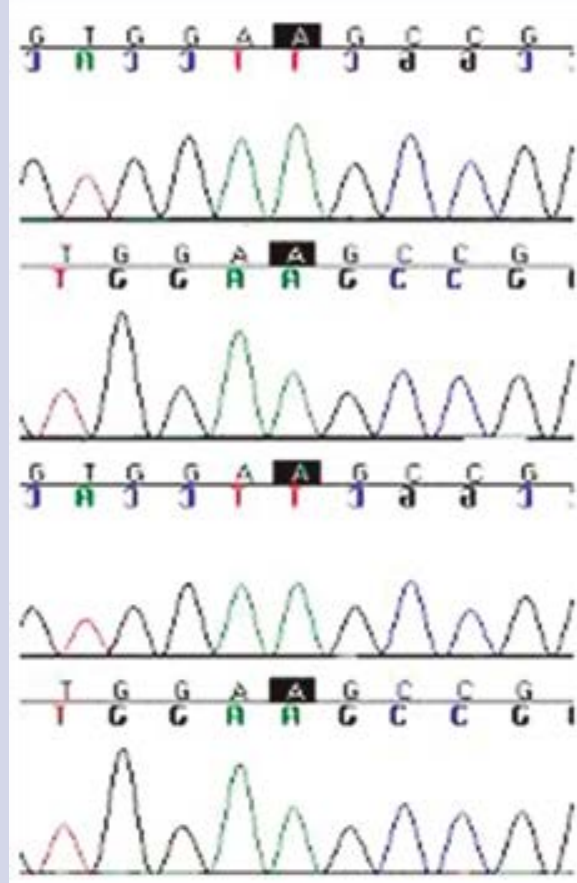

Mutación T489C
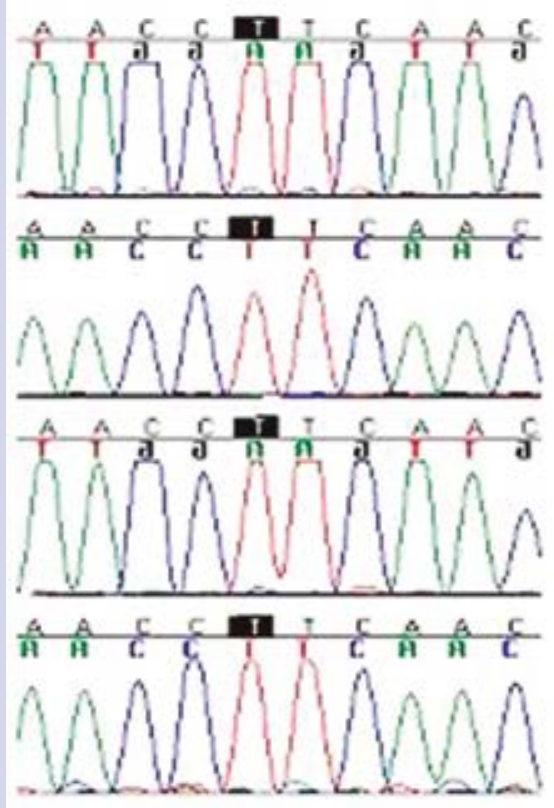

Mutación C12705T
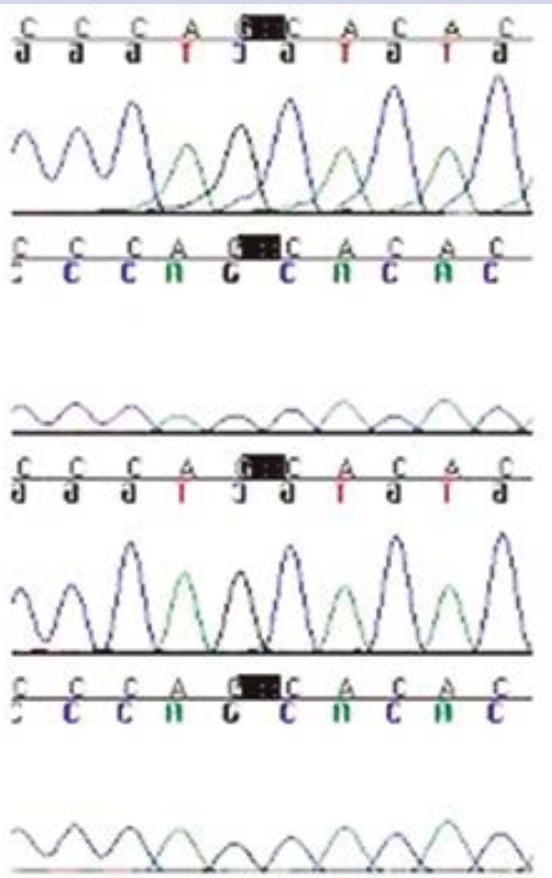

Mutación G6261A
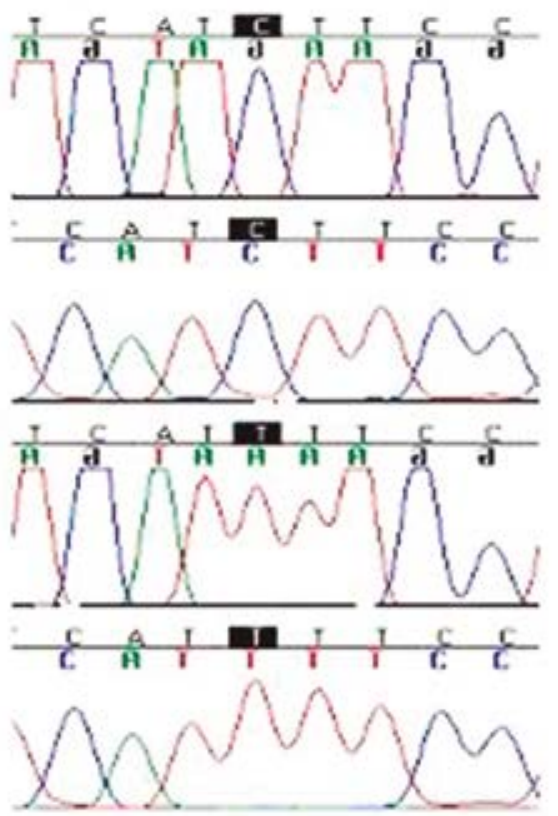

Mutación C16223T

Figura 1. Mutaciones localizadas experimentalmente, asociadas con cáncer de próstata en un paciente mexicano. 
estudiaron esta variación y afirmaron que ésta y otras más demuestran la existencia de patrones específicos de mutaciones somáticas de ADN mitocondrial en la evolución temprana del cáncer de próstata, lo que confirma la descripción de los resultados histopatológicos. ${ }^{18}$

Se utilizó la herramienta bioinformática Mitlmpact CSS Bioinformatics Lab para pronosticar la repercusión de las mutaciones G12009A, localizada en el locus MT-ND4 y C13478G en el locus MT-ND5, que incrementan la producción de especies reactivas de oxígeno (ROS) estrechamente vinculadas con la evolución del cáncer de próstata.

Los cambios estudiados en esta investigación en el ADN mitocondrial expresan un patrón de homoplasmia (mutación en todas las copias de ADN mitocondrial de la célula prostática). Hace poco se propuso que las mutaciones heteroplásmicas se encuentran en un estado primario del cáncer de próstata porque son decisivas para la supervivencia de la célula cancerígena; eventualmente, por señalizaciones moleculares se convierten en mutaciones homoplásmicas. ${ }^{19-22}$

En los tejidos maligno y sano es de particular interés la tasa de mutación somática porque quizá indique la pérdida de estabilidad del genoma mitocondrial, a pesar de los robustos mecanismos de reparación del ADN mitocondrial con orígenes nucleares, por lo que la mitocondria no es capaz de reparar el incremento de lesiones del ADN mitocondrial. ${ }^{23}$

Desde el punto de vista bioquímico, estos cambios son resultado de daños en los componentes de la cadena trasportadora de electrones, de manera que hay una pérdida significativa de energía expresada en ATP, lo que implica un error metabólico; sin embargo, para la célula maligna este proceso es un mecanismo de supervivencia que contribuye al desarrollo carcinogénico, siempre y cuando se supere el umbral de reparación mitocondrial. ${ }^{24}$

Con la base de datos MITOMAP y las secuencias del ADN mitocondrial humano (Homo sapiens sapiens), reportadas del GenBank del NCBI, como referencia, se identificó el haplogrupo A2 del paciente, a partir de los tejidos maligno y sano provenientes del haplogrupo $\mathrm{A}$, que se originó en Asia hace 30,000 a 50,000 años, y que predomina en Norteamérica septentrional. Es mayor en frecuencia, predomina en los mixtecas (73-93\%), mayas (53-84\%) y aztecas (65\%). El subclado A2 es típico de los pueblos indígenas americanos y de nativos del lejano oriente siberiano, sobre todo en chukchis y esquimales. ${ }^{25}$

En estudios previos se menciona que no existe influencia del haplogrupo en el cáncer de próstata. ${ }^{8-11}$ La mutación identificada y asociada con el cáncer de próstata, C12705T, corresponde al haplogrupo R, común en Euroasia. Varios autores han demostrado grandes diferencias con respecto al riesgo de cáncer de próstata entre los hombres de diferentes orígenes étnicos. ${ }^{12}$

Existe una gran variación geográfica en las frecuencias de haplogrupos, sobre todo en México, con gran diversidad de la población, determinadas por la mezcla de indígenas, europeos y africanos. En sus hallazgos, Kim y su grupo no encontraron asociación del riesgo de cáncer de próstata en la población americana. ${ }^{11}$ En México, para comprender plenamente la importancia de los estudios basados en haplogrupos poblacionales y el riesgo de cáncer de próstata debe establecerse una correlación exacta con la susceptibilidad a esta enfermedad con base en los marcadores ancestrales y los estudios filogeográficos.

En la urología clínica es muy común que el paciente tenga un resultado negativo en la biopsia transrectal; no obstante, después de 


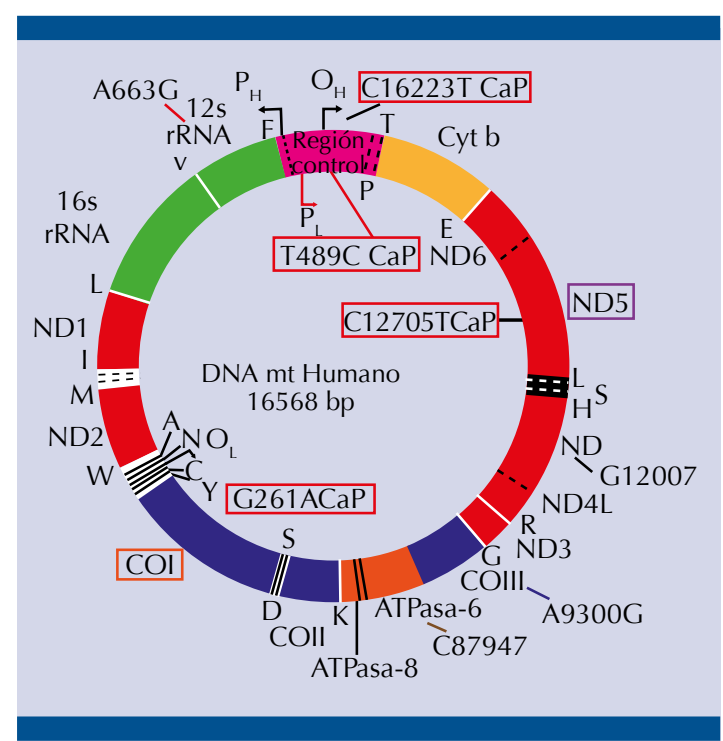

Figura 2. Mapa genético de ADNmt. Representación del genoma mitocondrial humano que indica las regiones y mutaciones localizadas experimentalmente asociadas al cáncer de próstata y otras patologías.

un seguimiento como medida de control, este diagnóstico es sustituido por el de cáncer de próstata metastásico. Lo importante es detectar oportunamente el cáncer de próstata valiéndose de la resonancia magnética por emisión de positrones (PET). Los estudios moleculares se aúnan a las técnicas clásicas de diagnóstico clínico tradicional del cáncer de próstata. ${ }^{26}$

\section{CONCLUSIONES}

Mediante la aplicación de técnicas moleculares, PCR punto final y secuenciación biparalela, se analizaron las mutaciones y polimorfismos del ADN mitocondrial (DNAmt) en un paciente mexicano con cáncer de próstata. Se identificaron las siguientes variaciones susceptibles al cáncer de próstata:T489A, G6261A, G12009A, C12705T, C13478G, C16223T, a partir de tejidos, maligno y sano obtenidos por resección transuretral de próstata; se examinó el comportamiento del cáncer de próstata en el genoma mitocondrial como futura herramienta complementaria al diagnóstico tradicional del cáncer de próstata.

\section{Financiamiento}

Los autores no recibieron ningún patrocinio para llevar a cabo este estudio/artículo

\section{Agradecimientos}

Este proyecto es apoyado por PAPIIT IN211413-3 fondo genético mitocondrial, actividad proteosomal y expresión génica en el cáncer de próstata en pacientes mexicanos.

\section{REFERENCIAS}

1. Estadísticas de México, INEGI, Obtenido el 03 de febrero de 2009 de http://www.inegi.org.mx/prod_serv/contenidos/espanol/bvinegi/productos/integracion/especiales/ mexmun/2008/MexMun08.pdf

2. Yeager M, Orr N, Hayes RB, et al. Genome-wide association study of prostate cancer identifies a second risk locus at 8q24. Nat. Genet. 2007;39: 645-649.

3. Shen X, Mizuguchi G, Hamiche A, et al. A chromatin remodeling complex involved in transcription and DNA processing. Nature 2000-406, 541-544.

4. Shutt TE, Shadel GS. A compendium of human mitochondrial gene expression machinery with links to disease. Environ. Mol. Mutagen. 2010;51:360-379.

5. Riemer J, Bulleid N, Herrmann J.M. Disulfide formation in the ER and mitochondria: two solutions to a common process. Science. 2009;324:1284-1287.

6. Soares P, Ermini L, Thomson N, Mormina M, Rito T, Röhl A, Salas A, Oppenheimer S, Macaulay V, Richards MB. Correcting for purifying selection: an improved human mitochondrial molecular clock. Am. J. Hum. Genet. 2009;84:740-759.

7. Parr RL, Dakubo GD, Thayer RE, McKenney K, BirchMachin MA. Mitochondrial DNA as a potential tool for early cancer detection. Hum. Genomics. 2006;2:252257

8. van Oven $M$, Kayser M. Updated comprehensive phylogenetic tree of global human mitochondrial DNA variation. Hum. Mutat. 2009;30:E386-E394.

9. Mosquera-Miguel A, Alvarez-lglesias V, Carracedo A, Salas A, Vega A, Carracedo A, Milne R, de León AC, Benitez J, Carracedo A, Salas A. Is mitochondrial DNA variation associated with sporadic breast cancer risk? Cancer Res. 2008;68:623-625. author reply 624

10. Mueller EE, Eder W, Mayr JA, Paulweber B, Sperl W, Horninger $\mathrm{W}$, Klocker $\mathrm{H}$, Kofler $\mathrm{B}$. Mitochondrial haplogroups 
and control region polymorphisms are not associated with prostate cancer in Middle European Caucasians. PLoS ONE. 2009;4:e6370

11. Kim W, Yoo TK, Shin DJ, Rho HW, Jin HJ, Kim ET, Bae YS. Mitochondrial DNA haplogroup analysis reveals no association between the common genetic lineages and prostate cancer in the Korean population. PLoS ONE. 2008;3:e2211.

12. Booker LM, Habermacher GM, Jessie BC, Sun QC, Baumann AK, Amin M, Lim SD, Fernandez-Golarz C, Lyles RH, Brown MD. North American white mitochondrial haplogroups in prostate and renal cancer. J. Urol. 2006; 175:468-472. discussion 472-473.

13. Khandrika L1, Kumar B, Koul S, et al. Oxidative stress in prostate cancer. Cancer Lett 2009.

14. Petros JA, Baumann AK, Ruiz-Pesini E, et al. MtDNA mutations increase tumorigenicity in prostate cancer. Proc Natl Acad Sci USA 2005; 102(3): 719-724.

15. Beardsle T. A War Not Won. Trends in Cancer Epidemiology. Sc. American. 1994. 118-125.

16. Booker LM, Habermacher GM, Jessie BC, et al. North American white mitochondrial haplogroups in prostate and renal cancer. J Urol 2006; 175(2):468-473.

17. Chan IS, Golden N, Greene GF, et al. Extensive somatic mitochondrial mutations in primary prostate cancer using laser capture microdissection. Cancer Res 2012. 62(22): 6470-6474.

18. Jerónimo C, Nomoto S, Caballero OL, et al. Mitochondrial mutations in early stage prostate cancer and bodily fluids. Oncogene 2001; 20(37): 5195-5198.
19. Liu SL, Lin X, Shi DY, et al. Reactive oxygen species stimulated human hepatoma cell proliferation via cross-talk between PI3-K/PKB and JNK signaling pathways. Arch Biochem Biophys 2012; 406(2): 173-182.

20. Ostronoff KL, Izquierdo JM, Enríquez JA, et al. Transient activation of mitochondrial translation regulates the expression of the mitochondrial genome during mammalian mitochondrial differentiation. Biochem J 1996 316:183-191.

21. Cook CC, Higuchi M. The awakening of an advanced malignant cancer: an insult to the mitochondrial genome. Biochim Biophys Acta 2012; 1820(5): 652-662.

22. Wallace DC. Diseases of the mitochondrial DNA. Annu Rev Biochem 1992; 61: 1175-1212.

23. Wang $Y$, Michikawa $Y$, Mallidis C., et al. Muscle-specific mutations accumulate with aging in critical human mtDNA control sites for replication, Proc Natl Acad Sci USA 2001; 98(7): 4022-4027.

24. Bardella C, Pollard PJ, Tomlinson I. SDH mutations in cancer. Biochim Biophys Acta 2011; 1807(11): 1432-1443.

25. Fagundes, Nelson J.R.; Ricardo Kanitz, Roberta Eckert, et al. Mitochondrial Population Genomics Supports a Single Pre-Clovis Origin with a Coastal Route for the Peopling of the Americas . American Journal of Human Genetics 82 (3): 583-592 (2008).

26. Fogg VC, Lanning NJ, Mackeigan JP. Mitochondria in cancer: at the crossroads of life and death 2011. Chin J Cancer; 30(8): 526-539.

\section{AVISO PARA LOS AUTORES}

Revista Mexicana de Urología tiene una nueva plataforma de gestión para envío de artículos. En: https://www.revisionporpares.com/index.php/RMUrol podrá inscribirse en nuestra base de datos administrada por el sistema Open Journal System (OJS) que ofrece las siguientes ventajas para los autores:

- Subir sus artículos directamente al sistema.

- Conocer, en cualquier momento, el estado de los artículos enviados, es decir, si ya fueron asignados a un revisor, aceptados con o sin cambios, o rechazados.

- Participar en el proceso editorial corrigiendo y modificando sus artículos hasta su aceptación final. 